\title{
Fixed Point Theorems for Contractions of Rational Type with PPF Dependence in Banach Spaces
}

\author{
J. Rocha, ${ }^{1}$ B. Rzepka, ${ }^{2}$ and K. Sadarangani ${ }^{1}$ \\ ${ }^{1}$ Departamento de Matemáticas, Universidad de Las Palmas de Gran Canaria, Campus de Tafira Baja, \\ 35017 Las Palmas de Gran Canaria, Spain \\ ${ }^{2}$ Department of Mathematics, Rzeszów University of Technology, Aleja Powstanców Warszawy 8, 35-959 Rzeszów, Poland
}

Correspondence should be addressed to B. Rzepka; brzepka@prz.edu.pl

Received 10 April 2014; Accepted 24 May 2014; Published 9 June 2014

Academic Editor: Józef Banaś

Copyright (c) 2014 J. Rocha et al. This is an open access article distributed under the Creative Commons Attribution License, which permits unrestricted use, distribution, and reproduction in any medium, provided the original work is properly cited.

We prove the existence of the PPF dependent fixed point in the Razumikhin class for contractions of rational type in Banach spaces, by using a general class of pairs of functions. Our result has as particular cases a great number of interesting consequences which extend and generalize some results appearing in the literature.

\section{Introduction}

Banach's contraction principle is one of the pivotal results of analysis. Its significance lies in its vast applicability to a great number of branches of mathematics and other sciences, for example, theory of existence of solutions for nonlinear differential, integral, and functional equations, variational inequalities, and optimization and approximation theory.

Generalizations of the contractive mapping theorem have been a heavily investigated branch of research. In particular, this principle was extended in [1], where the domain of the nonlinear operator involved is $\mathscr{C}([a, b], E)$ and the range is $E$, where $E$ is a Banach space. This result is known as the contraction theorem for operator with PPF (past, present, and future) dependence. The PPF fixed point theorems are useful for proving the existence of solutions for nonlinear functional-differential and integral equations which may depend upon the past history, present data, and future considerations. Some papers about fixed point theorems with PPF dependence have appeared in the literature (see, e.g., [15]).

On the other hand, Dass and Gupta in [6] and Jaggi in [7] were the pioneers in proving fixed point theorems using contractive conditions involving rational expressions. In [4], the authors present a fixed point theorem for contractions of rational type with PPF dependence.
The purpose of this paper is to present a fixed point theorem for generalized contractions of rational type with PPF dependence which has, as particular cases, interesting consequences. Particularly, our result extends the one appearing in [4].

\section{Preliminaries}

Throughout this paper, $E$ will denote a Banach space with norm $\|\cdot\|_{E}$ and $E_{0}=\mathscr{C}([a, b], E)$ will denote the space of the continuous $E$-valued functions defined on $[a, b]$ and equipped with the norm $\|\cdot\|_{E_{0}}$ given by

$$
\|\phi\|_{E_{0}}=\sup _{t \in[a, b]}\|\phi(t)\|_{E} \quad \text { for } \phi \in E_{0} .
$$

Let $T: E_{0} \rightarrow E$ be a mapping. A point $\phi \in E_{0}$ is said to be a PPF dependence fixed point of $T$ or a fixed point with PPF dependence of $T$ if $T \phi=\phi(c)$, for some $c \in[a, b]$.

For a fixed $c \in[a, b]$, the Razumikhin class $R_{c}$ is defined by

$$
R_{c}=\left\{\phi \in E_{0}:\|\phi\|_{E_{0}}=\|\phi(c)\|_{E}\right\} .
$$

Remark 1. Notice that, for $x \in E$ fixed, the function $\phi_{x}$, defined by

$$
\phi_{x}(t)=x \quad \text { for } t \in[a, b] \text {, }
$$


satisfies $\phi_{x} \in E_{0},\left\|\phi_{x}\right\|_{E_{0}}=\left\|\phi_{x}(c)\right\|_{E}=\|x\|$ for any $c \in[a, b]$ and, therefore, $\phi_{x} \in R_{c}$ for any $c \in[a, b]$. Consequently, $R_{c} \neq \emptyset$ for any $c \in[a, b]$.

We say that the class $R_{c}$ is algebraically closed with respect to difference if for any $\phi, \xi \in R_{c}$ we have $\phi-\xi \in R_{c}$. Similarly, we say that the class $R_{c}$ is topologically closed if it is closed with respect to the topology on $E_{0}$ induced by the norm $\|\cdot\|_{E_{0}}$.

The Razumikhin class plays an important role in the existence of PPF fixed point.

The first result about the existence of PPF fixed point appears in [1] and it is presented in the following theorem.

Theorem 2 (see [1]). Suppose that $T: E_{0} \rightarrow E$ is a mapping such that there exists $\alpha \in[0,1)$ satisfying

$$
\|T \phi-T \xi\|_{E} \leq \alpha\|\phi-\xi\|_{E_{0}}
$$

for any $\phi, \xi \in E_{0}$. If $R_{c}$ is topologically closed and algebraically closed with respect to difference for some $c \in[a, b]$, then $T$ has a unique PPF dependent fixed point in $R_{c}$.

Recently, in [4] the authors proved the following PPF dependent fixed point theorem for rational type contraction mappings.

Theorem 3 (see [4]). Let $T: E_{0} \rightarrow$ E be a mapping satisfying

$$
\|T \phi-T \xi\|_{E} \leq \alpha\|\phi-\xi\|_{E_{0}}+\beta \frac{\|\phi(c)-T \phi\|_{E}\|\xi(c)-T \xi\|_{E}}{1+\|T \phi-T \xi\|_{E}}
$$

for any $\phi, \xi \in E_{0}$ and where $\alpha, \beta \in[0,1)$ with $\alpha+\beta<1$ and $c \in[a, b]$. If $R_{c}$ is topologically closed and algebraically closed with respect to difference, then $T$ has a unique PPF dependent fixed point in $R_{c}$.

The main purpose of this paper is, by using a class of pairs of functions satisfying certain assumptions, to present new PPF dependent fixed point theorems for contractions of rational type. Particularly, our result generalizes the main result of [4] (Theorem 3).

\section{Main Results}

We start this section presenting the following class of pairs of functions $\mathfrak{F}$. A pair of functions $(\varphi, \phi)$ is said to belong to the class $\mathfrak{F}$ if they satisfy the following conditions:

(i) $\varphi, \phi:[0, \infty) \rightarrow[0, \infty)$;

(ii) for $t, s \in[0, \infty)$, if $\varphi(t) \leq \phi(s)$, then $t \leq s$;

(iii) for $\left(t_{n}\right)$ and $\left(s_{n}\right)$ sequences in $[0, \infty)$ such that

$$
\lim _{n \rightarrow \infty} t_{n}=\lim _{n \rightarrow \infty} s_{n}=a,
$$

if $\varphi\left(t_{n}\right) \leq \phi\left(s_{n}\right)$ for any $n \in \mathbb{N}$, then $a=0$.
Remark 4. Notice that if $(\varphi, \phi) \in \mathfrak{F}$ and $\varphi(t) \leq \phi(t)$, then $t=0$, since we can take $t_{n}=s_{n}=t$ for any $n \in \mathbb{N}$ and by (iii) we deduce $t=0$.

In the sequel, we present some interesting examples of pairs of functions belonging to the class $\mathfrak{F}$ which will be very important in our study.

Example 5. Let $\varphi:[0, \infty) \rightarrow[0, \infty)$ be a continuous and increasing function such that $\varphi(t)=0$ if and only if $t=0$ (these functions are known in the literature as altering distance functions).

Let $\phi:[0, \infty) \rightarrow[0, \infty)$ be a nondecreasing function such that $\phi(t)=0$ if and only if $t=0$ and suppose that $\phi \leq \varphi$.

Then the pair $(\varphi, \varphi-\phi) \in \mathfrak{F}$.

In fact, it is clear that $(\varphi, \varphi-\phi)$ satisfy (i).

To prove (ii), suppose that $t, s \in[0, \infty)$ and $\varphi(t) \leq(\varphi-$ $\phi)(s)$. Then, from

$$
\varphi(t) \leq \varphi(s)-\phi(s) \leq \varphi(s)
$$

and taking into account the increasing character of $\varphi$, we can deduce that $t \leq s$.

In order to prove (iii), we suppose that

$$
\varphi\left(t_{n}\right) \leq \varphi\left(s_{n}\right)-\phi\left(s_{n}\right) \leq \varphi\left(s_{n}\right) \quad \text { for any } n \in \mathbb{N},
$$

where $t_{n}, s_{n} \in[0, \infty)$ and

$$
\lim _{n \rightarrow \infty} t_{n}=\lim _{n \rightarrow \infty} s_{n}=a .
$$

Taking $n \rightarrow \infty$ in (8), we infer that $\lim _{n \rightarrow \infty} \phi\left(s_{n}\right)=0$.

Let us suppose that $a>0$. Since $\lim _{n \rightarrow \infty} s_{n}=a>0$, we can find $\varepsilon>0$ and a subsequence $\left(s_{n_{k}}\right)$ of $\left(s_{n}\right)$ such that $s_{n_{k}}>\varepsilon$ for any $k \in \mathbb{N}$. As $\phi$ is nondecreasing, we have $\phi\left(s_{n_{k}}\right)>\phi(\varepsilon)$ for any $k \in \mathbb{N}$ and, consequently, $\lim _{k \rightarrow \infty} \phi\left(s_{n_{k}}\right) \geq \phi(\varepsilon)$. This contradicts the fact that $\lim _{k \rightarrow \infty} \phi\left(s_{n_{k}}\right)=0$. Therefore, $a=0$.

This proves that $(\varphi, \varphi-\phi) \in \mathfrak{F}$.

An interesting particular case is when $\varphi$ is the identity mapping, $\varphi=1_{[0, \infty)}$, and $\phi:[0, \infty) \rightarrow[0, \infty)$ is a nondecreasing function such that $\phi(t)=0$ if and only if $t=0$ and $\phi(t) \leq t$ for any $t \in[0, \infty)$.

Example 6. Let $S$ be the class of functions defined by

$$
S=\left\{\alpha:[0, \infty) \longrightarrow[0,1):\left\{\alpha\left(t_{n}\right) \longrightarrow 1 \Longrightarrow t_{n} \longrightarrow 0\right\}\right\} .
$$

Let us consider the pairs of functions $\left(1_{[0, \infty)}, \alpha 1_{[0, \infty)}\right)$, where $\alpha \in S$ and $\alpha 1_{[0, \infty)}$ is defined by $\left(\alpha 1_{[0, \infty)}\right)(t)=\alpha(t) t$, for $t \epsilon$ $[0, \infty)$.

Then $\left(1_{[0, \infty)}, \alpha 1_{[0, \infty)}\right) \in \mathfrak{F}$.

It is clear that the pairs $\left(1_{[0, \infty)}, \alpha 1_{[0, \infty)}\right)$ with $\alpha \in S$ satisfy (i).

To prove (ii), from

$$
1_{[0, \infty)}(t) \leq\left(\alpha 1_{[0, \infty)}\right)(s) \quad \text { for } t, s \in[0, \infty)
$$

we infer, since $\alpha:[0, \infty) \rightarrow[0,1)$, that

$$
t \leq \alpha(s) s<s
$$

and, consequently, $\left(1_{[0, \infty)}, \alpha 1_{[0, \infty)}\right)$ satisfies (ii). 
In order to prove (iii), we suppose that

$$
1_{[0, \infty)}\left(t_{n}\right)=t_{n} \leq\left(\alpha 1_{[0, \infty)}\right)\left(s_{n}\right)=\alpha\left(s_{n}\right) s_{n} \quad \text { for any } n \in \mathbb{N},
$$

where $t_{n}, s_{n} \in[0, \infty)$ and $\lim _{n \rightarrow \infty} t_{n}=\lim _{n \rightarrow \infty} s_{n}=a$.

Let us suppose that $a>0$.

Since $\lim _{n \rightarrow \infty} s_{n}=a>0$, we can find a subsequence $\left(s_{n_{k}}\right)$ such that $s_{n_{k}}>0$ for any $k \in \mathbb{N}$. Now, as

$$
t_{n} \leq \alpha\left(s_{n}\right) s_{n} \leq s_{n} \quad \text { for any } n \in \mathbb{N}
$$

in particular, we have

$$
t_{n_{k}} \leq \alpha\left(s_{n_{k}}\right) s_{n_{k}} \leq s_{n_{k}} \quad \text { for any } k \in \mathbb{N}
$$

and, since $s_{n_{k}}>0$ for any $k \in \mathbb{N}$,

$$
\frac{t_{n_{k}}}{s_{n_{k}}} \leq \alpha\left(s_{n_{k}}\right) \leq 1 .
$$

Taking $k \rightarrow \infty$ in the last inequality, we obtain

$$
\lim _{k \rightarrow \infty} \alpha\left(s_{n_{k}}\right)=1
$$

Finally, since $\alpha \in S$, we infer that $\lim _{k \rightarrow \infty} s_{n_{k}}=0$ and this contradicts the fact that $\lim _{n \rightarrow \infty} s_{n}=a>0$.

Therefore, $a=0$.

This proves that $\left(1_{[0, \infty)}, \alpha 1_{[0, \infty)}\right) \in \mathfrak{F}$ for $\alpha \in S$.

Remark 7. Suppose that $g:[0, \infty) \rightarrow[0, \infty)$ is an increasing function and $(\varphi, \phi) \in \mathfrak{F}$. Then it is easily seen that the pair $(g \circ \varphi, g \circ \phi) \in \mathfrak{F}$.

Now, we are ready to present our main result.

Theorem 8. Let $T: E_{0} \rightarrow E$ be a mapping such that there exists a pair of functions $(\varphi, \phi) \in \mathfrak{F}$ and $c \in[a, b]$ such that

$$
\begin{aligned}
\varphi\left(\|T \xi-T \eta\|_{E}\right) \\
\leq \max \left\{\phi\left(\|\xi-\eta\|_{E_{0}}\right),\right. \\
\left.\phi\left(\frac{\|\xi(c)-T \xi\|_{E} \cdot\|\eta(c)-T \eta\|_{E}}{1+\|T \xi-T \eta\|_{E}}\right)\right\}
\end{aligned}
$$

for any $\xi, \eta \in E_{0}$. If $R_{c}$ is topologically closed and algebraically closed with respect to difference, then $T$ has a unique PPF dependent fixed point in $R_{c}$.

Proof. Let $\xi_{0}$ be an arbitrary function in $R_{c}$ (whose existence is guaranteed by Remark 1). Since $\xi_{0} \in R_{c} \subset E_{0}$, put $x_{1}=$ $T \xi_{0} \in E$.

Again, by Remark 1, we can find $\xi_{1} \in R_{c}$ such that

$$
T \xi_{0}=x_{1}=\xi_{1}(c) \text {. }
$$

Since $\xi_{1} \in R_{c} \subset E_{0}$, put $x_{2}=T \xi_{1} \in E$. Using the same argument, we can find $\xi_{2} \in R_{c}$ such that

$$
T \xi_{1}=x_{2}=\xi_{2}(c) \text {. }
$$

Repeating this process, we can obtain a sequence $\left(\xi_{n}\right)$ in $R_{c}$ such that

$$
T \xi_{n-1}=\xi_{n}(c) \quad \text { for any } n \in \mathbb{N} .
$$

Since $R_{c}$ is algebraically closed with respect to difference, we have

$$
\left\|\xi_{p}-\xi_{q}\right\|_{E_{0}}=\left\|\xi_{p}(c)-\xi_{q}(c)\right\|_{E}
$$

for any $p, q \in \mathbb{N}$.

First, we will prove that $\lim _{n \rightarrow \infty}\left\|\xi_{n+1}-\xi_{n}\right\|_{E_{0}}=0$. In fact, taking into account (21) and (22), we get

$$
\begin{aligned}
\left\|\xi_{n+1}-\xi_{n}\right\|_{E_{0}} & =\left\|\xi_{n+1}(c)-\xi_{n}(c)\right\|_{E} \\
& =\left\|T \xi_{n}-T \xi_{n-1}\right\|_{E} \text { for any } n \in \mathbb{N}
\end{aligned}
$$

and, therefore, applying the contractive condition, we have

$$
\begin{aligned}
\varphi( & \left.\left\|\xi_{n+1}-\xi_{n}\right\|_{E_{0}}\right) \\
= & \varphi\left(\left\|T \xi_{n}-T \xi_{n-1}\right\|_{E}\right) \\
\leq \max & \left\{\phi\left(\left\|\xi_{n}-\xi_{n-1}\right\|_{E_{0}}\right),\right. \\
& \left.\phi\left(\frac{\left\|\xi_{n}(c)-T \xi_{n}\right\|_{E} \cdot\left\|\xi_{n-1}(c)-T \xi_{n-1}\right\|_{E}}{1+\left\|T \xi_{n}-T \xi_{n-1}\right\|_{E}}\right)\right\} .
\end{aligned}
$$

Let us suppose that there exists $n_{0} \in \mathbb{N}$ such that $\| \xi_{n_{0}+1}-$ $\xi_{n_{0}} \|_{E_{0}}=0$.

In this case, $\xi_{n_{0}+1}=\xi_{n_{0}}$ and, consequently, $\xi_{n_{0}+1}(c)=$ $\xi_{n_{0}}(c)$.

By (21), we have

$$
T \xi_{n_{0}}=\xi_{n_{0}+1}(c)=\xi_{n_{0}}(c)
$$

and $\xi_{n_{0}}$ would be the PPF dependent fixed point. $\mathbb{N}$.

In the sequel we suppose that $\left\|\xi_{n+1}-\xi_{n}\right\|_{E_{0}} \neq 0$ for any $n \epsilon$

Now, we can distinguish two cases.

Case 1. Consider

$$
\begin{aligned}
\max \left\{\phi\left(\left\|\xi_{n}-\xi_{n-1}\right\|_{E_{0}}\right),\right. & \\
& \left.\phi\left(\frac{\left\|\xi_{n}(c)-T \xi_{n}\right\|_{E} \cdot\left\|\xi_{n-1}(c)-T \xi_{n-1}\right\|_{E}}{1+\left\|T \xi_{n}-T \xi_{n-1}\right\|_{E}}\right)\right\} \\
= & \phi\left(\left\|\xi_{n}-\xi_{n-1}\right\|_{E_{0}}\right) .
\end{aligned}
$$

In this case, from (24), we infer

$$
\varphi\left(\left\|\xi_{n+1}-\xi_{n}\right\|_{E_{0}}\right) \leq \phi\left(\left\|\xi_{n}-\xi_{n-1}\right\|_{E_{0}}\right)
$$

and, since $(\varphi, \phi) \in \mathfrak{F}$, we deduce

$$
\left\|\xi_{n+1}-\xi_{n}\right\|_{E_{0}} \leq\left\|\xi_{n}-\xi_{n-1}\right\|_{E_{0}} .
$$


Case 2. Consider

$$
\begin{aligned}
\max & \left\{\phi\left(\left\|\xi_{n}-\xi_{n-1}\right\|_{E_{0}}\right),\right. \\
& \left.\phi\left(\frac{\left\|\xi_{n}(c)-T \xi_{n}\right\|_{E} \cdot\left\|\xi_{n-1}(c)-T \xi_{n-1}\right\|_{E}}{1+\left\|T \xi_{n}-T \xi_{n-1}\right\|_{E}}\right)\right\} \\
= & \phi\left(\frac{\left\|\xi_{n}(c)-T \xi_{n}\right\|_{E} \cdot\left\|\xi_{n-1}(c)-T \xi_{n-1}\right\|_{E}}{1+\left\|T \xi_{n}-T \xi_{n-1}\right\|_{E}}\right) .
\end{aligned}
$$

In this case, from $(24)$ and since $(\varphi, \phi) \in \mathfrak{F}$, we infer

$$
\left\|\xi_{n+1}-\xi_{n}\right\|_{E_{0}} \leq \frac{\left\|\xi_{n}(c)-T \xi_{n}\right\|_{E} \cdot\left\|\xi_{n-1}(c)-T \xi_{n-1}\right\|_{E}}{1+\left\|T \xi_{n}-T \xi_{n-1}\right\|_{E}}
$$

By (21) and (22), we have

$$
\begin{aligned}
\left\|\xi_{n+1}-\xi_{n}\right\|_{E_{0}} & \leq \frac{\left\|\xi_{n}(c)-\xi_{n+1}(c)\right\|_{E} \cdot\left\|\xi_{n-1}(c)-\xi_{n}(c)\right\|_{E}}{1+\left\|\xi_{n+1}(c)-\xi_{n}(c)\right\|_{E}} \\
& =\frac{\left\|\xi_{n+1}-\xi_{n}\right\|_{E_{0}} \cdot\left\|\xi_{n}-\xi_{n-1}\right\|_{E_{0}}}{1+\left\|\xi_{n+1}-\xi_{n}\right\|_{E_{0}}} .
\end{aligned}
$$

Since $\left\|\xi_{n+1}-\xi_{n}\right\|_{E_{0}} \neq 0$, from the last inequality, it follows that

$$
1 \leq \frac{\left\|\xi_{n}-\xi_{n-1}\right\|_{E_{0}}}{1+\left\|\xi_{n+1}-\xi_{n}\right\|_{E_{0}}}
$$

and, therefore,

$$
\left\|\xi_{n+1}-\xi_{n}\right\|_{E_{0}}<1+\left\|\xi_{n+1}-\xi_{n}\right\|_{E_{0}} \leq\left\|\xi_{n}-\xi_{n-1}\right\|_{E_{0}} .
$$

In both cases, we obtain that inequality (28) is satisfied and, consequently, the sequence $\left(\left\|\xi_{n+1}-\xi_{n}\right\|_{E_{0}}\right)$ is a decreasing sequence of nonnegative real numbers.

Put $r=\lim _{n \rightarrow \infty}\left\|\xi_{n+1}-\xi_{n}\right\|_{E_{0}}$, where $r \geq 0$, and denote

$$
\begin{aligned}
& A=\{n \in \mathbb{N}: n \text { satisfies (26) }\}, \\
& B=\{n \in \mathbb{N}: n \text { satisfies (29) }\} .
\end{aligned}
$$

We remark the following.

(1) If Card $A=\infty$, then from (24) we can find infinitely many natural numbers $n$ satisfying inequality (27) and, since $\lim _{n \rightarrow \infty}\left\|\xi_{n+1}-\xi_{n}\right\|_{E_{0}}=$ $\lim _{n \rightarrow \infty}\left\|\xi_{n}-\xi_{n-1}\right\|_{E_{0}}=r$ and $(\varphi, \phi) \in \mathfrak{F}$, we deduce that $r=0$.

(2) If Card $B=\infty$, then from (24) we can find infinitely many $n \in \mathbb{N}$ such that

$$
\begin{aligned}
& \varphi\left(\left\|\xi_{n+1}-\xi_{n}\right\|_{E_{0}}\right) \\
& \quad \leq \phi\left(\frac{\left\|\xi_{n}(c)-T \xi_{n}\right\|_{E} \cdot\left\|\xi_{n-1}(c)-T \xi_{n-1}\right\|_{E}}{1+\left\|T \xi_{n}-T \xi_{n-1}\right\|_{E}}\right) .
\end{aligned}
$$

Since $(\varphi, \phi) \in \mathfrak{F}$ and using a similar argument to the one used in Case 2, we obtain

$$
\left\|\xi_{n+1}-\xi_{n}\right\|_{E_{0}} \leq \frac{\left\|\xi_{n+1}-\xi_{n}\right\|_{E_{0}} \cdot\left\|\xi_{n}-\xi_{n-1}\right\|_{E_{0}}}{1+\left\|\xi_{n+1}-\xi_{n}\right\|_{E_{0}}}
$$

for infinitely many $n \in \mathbb{N}$. Taking $n \rightarrow \infty$ in the last inequality and taking into account that $\lim _{n \rightarrow \infty}\left\|\xi_{n+1}-\xi_{n}\right\|_{E_{0}}=r$ we deduce

$$
r \leq \frac{r^{2}}{1+r}
$$

and, consequently, $r \leq 0$. Since $r \geq 0$, we obtain $r=0$.

Therefore,

$$
\lim _{n \rightarrow \infty}\left\|\xi_{n+1}-\xi_{n}\right\|_{E_{0}}=0
$$

Next, we will prove that $\left(\xi_{n}\right)$ is a Cauchy sequence in $E_{0}$. In contrary case, since $\lim _{n \rightarrow \infty}\left\|\xi_{n+1}-\xi_{n}\right\|_{E_{0}}=0$, by Lemma 2.1 of [8], we can find $\varepsilon>0$ and subsequences $\left(\xi_{n(k)}\right)$ and $\left(\xi_{m(k)}\right)$ of $\left(\xi_{n}\right)$ satisfying

(i) $n(k)>m(k) \geq k$ for $k>0$,

(ii) $\varepsilon \leq\left\|\xi_{n(k)}-\xi_{m(k)}\right\|_{E_{0}}, \quad\left\|\xi_{n(k)-1}-\xi_{m(k)}\right\|_{E_{0}}<\varepsilon$ for $k>0$,

(iii) $\lim _{k \rightarrow \infty}\left\|\xi_{n(k)}-\xi_{m(k)}\right\|_{E_{0}}=\lim _{k \rightarrow \infty}\left\|\xi_{n(k)}-\xi_{m(k)+1}\right\|_{E_{0}}$

$$
\begin{aligned}
& =\lim _{k \rightarrow \infty}\left\|\xi_{n(k)+1}-\xi_{m(k)}\right\|_{E_{0}} \\
& =\lim _{k \rightarrow \infty}\left\|\xi_{n(k)+1}-\xi_{m(k)+1}\right\|_{E_{0}}=\varepsilon .
\end{aligned}
$$

Since $\xi_{n(k)+1}, \xi_{m(k)+1} \in R_{c}$ for any $k \in \mathbb{N}$, from (21) and (22), we have

$$
\begin{aligned}
\left\|\xi_{n(k)+1}-\xi_{m(k)+1}\right\|_{E_{0}} & =\left\|\xi_{n(k)+1}(c)-\xi_{m(k)+1}(c)\right\|_{E} \\
& =\left\|T \xi_{n(k)}-T \xi_{m(k)}\right\|_{E}
\end{aligned}
$$

for any $k \in \mathbb{N}$.

Using the contractive condition and (21) and (22), we obtain

$$
\begin{aligned}
& \varphi(\left.\left\|\xi_{n(k)+1}-\xi_{m(k)+1}\right\|_{E_{0}}\right) \\
&= \varphi\left(\left\|T \xi_{n(k)}-T \xi_{m(k)}\right\|_{E}\right) \\
& \leq \max \left\{\phi\left(\left\|\xi_{n(k)}-\xi_{m(k)}\right\|_{E_{0}}\right),\right. \\
&\left.\phi\left(\frac{\left\|\xi_{n(k)}(c)-T \xi_{n(k)}\right\|_{E} \cdot\left\|\xi_{m(k)}(c)-T \xi_{m(k)}\right\|_{E}}{1+\left\|T \xi_{n(k)}-T \xi_{m(k)}\right\|_{E}}\right)\right\}
\end{aligned}
$$




$$
\begin{aligned}
&=\max \{ \phi\left(\left\|\xi_{n(k)}-\xi_{m(k)}\right\|_{E_{0}}\right), \\
& \phi\left(\left\|\xi_{n(k)}(c)-\xi_{n(k)+1}(c)\right\|_{E}\right. \\
&\left.\left\|\xi_{m(k)}(c)-\xi_{m(k)+1}(c)\right\|_{E}\right) \\
& \times\left.\left.\left(1+\left\|\xi_{n(k)+1}(c)-\xi_{m(k)+1}(c)\right\|_{E}\right)^{-1}\right)\right\} \\
&=\max \{\phi\left(\left\|\xi_{n(k)}-\xi_{m(k)}\right\|_{E_{0}}\right), \\
& \phi(\left(\left\|\xi_{n(k)+1}-\xi_{n(k)}\right\|_{E_{0}} \cdot\left\|\xi_{m(k)+1}-\xi_{m(k)}\right\|_{E_{0}}\right) \\
&\left.\left.\times\left(1+\left\|\xi_{n(k)+1}-\xi_{m(k)+1}\right\|_{E_{0}}\right)^{-1}\right)\right\}
\end{aligned}
$$

for any $k \in \mathbb{N}$.

Let us put

$$
\begin{aligned}
C=\{ & \{k \in \mathbb{N}: \\
& \left.\varphi\left(\left\|\xi_{n(k)+1}-\xi_{m(k)+1}\right\|_{E_{0}}\right) \leq \phi\left(\left\|\xi_{n(k)}-\xi_{m(k)}\right\|_{E_{0}}\right)\right\}, \\
D=\{k \in \mathbb{N}: & \\
& \varphi\left(\left\|\xi_{n(k)+1}-\xi_{m(k)+1}\right\|_{E_{0}}\right) \\
\leq & \left.\phi\left(\frac{\left\|\xi_{n(k)+1}-\xi_{n(k)}\right\|_{E_{0}} \cdot\left\|\xi_{m(k)+1}-\xi_{m(k)}\right\|_{E_{0}}}{1+\left\|\xi_{n(k)+1}-\xi_{m(k)+1}\right\|_{E_{0}}}\right)\right\} .
\end{aligned}
$$

By (41) we have Card $C=\infty$ or $\operatorname{Card} D=\infty$.

Let us suppose that $\operatorname{Card} C=\infty$. Then there exist infinitely many $k \in \mathbb{N}$ such that

$$
\varphi\left(\left\|\xi_{n(k)+1}-\xi_{m(k)+1}\right\|_{E_{0}}\right) \leq \phi\left(\left\|\xi_{n(k)}-\xi_{m(k)}\right\|_{E_{0}}\right)
$$

and since $(\varphi, \phi) \in \mathfrak{F}$ and

$$
\lim _{k \rightarrow \infty}\left\|\xi_{n(k)+1}-\xi_{m(k)+1}\right\|_{E_{0}}=\lim _{k \rightarrow \infty}\left\|\xi_{n(k)}-\xi_{m(k)}\right\|_{E_{0}}=\varepsilon
$$

we infer from (39) that $\varepsilon=0$. This is a contradiction.

Let us suppose that $\operatorname{Card} D=\infty$. In this case, we can find infinitely many $k \in \mathbb{N}$ such that

$$
\begin{aligned}
\varphi & \left(\left\|\xi_{n(k)+1}-\xi_{m(k)+1}\right\|_{E_{0}}\right) \\
& \leq \phi\left(\frac{\left\|\xi_{n(k)+1}-\xi_{n(k)}\right\|_{E_{0}} \cdot\left\|\xi_{m(k)+1}-\xi_{m(k)}\right\|_{E_{0}}}{1+\left\|\xi_{n(k)+1}-\xi_{m(k)+1}\right\|_{E_{0}}}\right)
\end{aligned}
$$

and since $(\varphi, \phi) \in \mathfrak{F}$, we infer

$$
\begin{aligned}
& \left\|\xi_{n(k)+1}-\xi_{m(k)+1}\right\|_{E_{0}} \\
& \quad \leq \frac{\left\|\xi_{n(k)+1}-\xi_{n(k)}\right\|_{E_{0}} \cdot\left\|\xi_{m(k)+1}-\xi_{m(k)}\right\|_{E_{0}}}{1+\left\|\xi_{n(k)+1}-\xi_{m(k)+1}\right\|_{E_{0}}} .
\end{aligned}
$$

Taking $k \rightarrow \infty$ and in view of (38) and (39), it follows that $\varepsilon \leq 0$ and this is a contradiction.

Therefore, since in both possibilities Card $C=\infty$ and Card $D=\infty$ we obtain a contradiction, we deduce that $\left(\xi_{n}\right)$ is a Cauchy sequence in $E_{0}$.

Since $E_{0}$ is a Banach space, we can find $\xi^{*} \in E_{0}$ such that $\lim _{n \rightarrow \infty} \xi_{n}=\xi^{*}$. As $\xi_{n} \in R_{c}$ and $R_{c}$ is topologically closed, we have $\xi^{*} \in R_{c}$.

Next, we will prove that $\xi^{*}$ is a PPF dependent fixed point of $T$. In fact, by the contractive condition, we obtain

$$
\begin{aligned}
& \varphi\left(\left\|T \xi^{*}-T \xi_{n}\right\|_{E}\right) \\
& \leq \max \left\{\phi\left(\left\|\xi^{*}-\xi_{n}\right\|_{E_{0}}\right),\right. \\
&\left.\phi\left(\frac{\left\|\xi^{*}(c)-T \xi^{*}\right\|_{E} \cdot\left\|\xi_{n}(c)-T \xi_{n}\right\|_{E}}{1+\left\|T \xi^{*}-T \xi_{n}\right\|_{E}}\right)\right\}
\end{aligned}
$$

for any $n \in \mathbb{N}$.

We can distinguish two cases again.

(1) There exist infinitely many $n \in \mathbb{N}$ such that

$$
\varphi\left(\left\|T \xi^{*}-T \xi_{n}\right\|_{E}\right) \leq \phi\left(\left\|\xi^{*}-\xi_{n}\right\|_{E_{0}}\right) .
$$

In this case, since $(\varphi, \phi) \in \mathfrak{F}$, we obtain

$$
\left\|T \xi^{*}-T \xi_{n}\right\|_{E} \leq\left\|\xi^{*}-\xi_{n}\right\|_{E_{0}}
$$

for infinitely many $n \in \mathbb{N}$. Since $\lim _{n \rightarrow \infty} \xi_{n}=\xi^{*}$, taking $n \rightarrow \infty$ in the last inequality, we obtain

$$
\lim _{n \rightarrow \infty} T \xi_{n}=T \xi^{*}
$$

where, to simplify our considerations, we will denote the subsequence by the same symbol $\left(T \xi_{n}\right)$. By (21),

$$
T \xi^{*}=\lim _{n \rightarrow \infty} T \xi_{n}=\lim _{n \rightarrow \infty} \xi_{n+1}(c) .
$$

$\xi_{n} \rightarrow \xi^{*}$ in $E_{0}$; this means that

$$
\sup _{t \in[a, b]}\left\|\xi_{n}(t)-\xi^{*}(t)\right\|_{E} \longrightarrow 0
$$

and, consequently, $\lim _{n \rightarrow \infty} \xi_{n+1}(c)=\xi^{*}(c)$. From this last result and from (51) we deduce that

$$
T \xi^{*}=\xi^{*}(c)
$$

and, therefore, $\xi^{*}$ is a PPF dependent fixed point of $T$ in $R_{c}$.

(2) There exist infinitely many $n \in \mathbb{N}$ such that

$$
\varphi\left(\left\|T \xi^{*}-T \xi_{n}\right\|_{E}\right) \leq \phi\left(\frac{\left\|\xi^{*}(c)-T \xi^{*}\right\|_{E} \cdot\left\|\xi_{n}(c)-T \xi_{n}\right\|_{E}}{1+\left\|T \xi^{*}-T \xi_{n}\right\|_{E}}\right) .
$$


To simplify our considerations, we will denote the subsequence by the same symbol $\left(T \xi_{n}\right)$. Since $(\varphi, \phi) \in$ $\mathfrak{F}$, we infer

$$
\left\|T \xi^{*}-T \xi_{n}\right\|_{E} \leq \frac{\left\|\xi^{*}(c)-T \xi^{*}\right\|_{E} \cdot\left\|\xi_{n}(c)-T \xi_{n}\right\|_{E}}{1+\left\|T \xi^{*}-T \xi_{n}\right\|_{E}}
$$

for any $n \in \mathbb{N}$. Using (21), we have that $T \xi_{n}=\xi_{n+1}(c)$ and, therefore,

$$
\left\|T \xi^{*}-T \xi_{n}\right\|_{E} \leq \frac{\left\|\xi^{*}(c)-T \xi^{*}\right\|_{E} \cdot\left\|\xi_{n}(c)-\xi_{n+1}(c)\right\|_{E}}{1+\left\|T \xi^{*}-T \xi_{n}\right\|_{E}}
$$

for any $n \in \mathbb{N}$. Taking $n \rightarrow \infty$ and by (38) since $\lim _{n \rightarrow \infty}\left\|\xi_{n}(c)-\xi_{n+1}(c)\right\|_{E}=0$, we infer (50). From the above case, we deduce that $\xi^{*}$ is a PPF dependent fixed point of $T$ in $R_{c}$.

Therefore, we have proved that in both cases $\xi^{*}$ is a PPF dependent fixed point of $T$ in $R_{c}$.

Finally, we will prove the uniqueness of PPF dependent fixed point of $T$ in $R_{c}$.

Suppose that $\phi^{*}$ is another PPF dependent fixed point of $T$ in $R_{c}$. Then, since $\xi^{*}, \phi^{*} \in R_{c}$ and $R_{c}$ is algebraically closed, we obtain

$$
\left\|\xi^{*}-\phi^{*}\right\|_{E_{0}}=\left\|\xi^{*}(c)-\phi^{*}(c)\right\|_{E^{*}}
$$

As $\xi^{*}(c)=T \xi^{*}$ and $\phi^{*}(c)=T \phi^{*}$ because $\xi^{*}$ and $\phi^{*}$ are PPF dependent fixed points of $T$, we infer

$$
\left\|\xi^{*}-\phi^{*}\right\|_{E_{0}}=\left\|\xi^{*}(c)-\phi^{*}(c)\right\|_{E}=\left\|T \xi^{*}-T \phi^{*}\right\|_{E^{*}}
$$

Consequently, using the contractive condition, we get

$$
\begin{aligned}
& \varphi\left(\left\|\xi^{*}-\phi^{*}\right\|_{E_{0}}\right) \\
& =\varphi\left(\left\|T \xi^{*}-T \phi^{*}\right\|_{E}\right) \\
& \leq \max \left\{\phi\left(\left\|\xi^{*}-\phi^{*}\right\|_{E_{0}}\right),\right. \\
& \left.\phi\left(\frac{\left\|\xi^{*}(c)-T \xi^{*}\right\|_{E} \cdot\left\|\phi^{*}(c)-T \phi^{*}\right\|_{E}}{1+\left\|T \xi^{*}-T \phi^{*}\right\|_{E}}\right)\right\} \\
& =\max \left\{\phi\left(\left\|\xi^{*}-\phi^{*}\right\|_{E_{0}}\right), \phi(0)\right\} .
\end{aligned}
$$

We can distinguish two cases.

(i) Consider $\max \left\{\phi\left(\left\|\xi^{*}-\phi^{*}\right\|_{E_{0}}\right), \phi(0)\right\}=\phi\left(\| \xi^{*}-\right.$ $\left.\phi^{*} \|_{E_{0}}\right)$. In this case, from (59) we have

$$
\varphi\left(\left\|\xi^{*}-\phi^{*}\right\|_{E_{0}}\right) \leq \phi\left(\left\|\xi^{*}-\phi^{*}\right\|_{E_{0}}\right)
$$

Now, since $(\varphi, \phi) \in \mathfrak{F}$ and using Remark 4, we get $\left\|\xi^{*}-\phi^{*}\right\|_{E_{0}}=0$ and, therefore, $\xi^{*}=\phi^{*}$. (ii) Consider $\max \left\{\phi\left(\left\|\xi^{*}-\phi^{*}\right\|_{E_{0}}\right), \phi(0)\right\}=\phi(0)$. From (59) we obtain

$$
\varphi\left(\left\|\xi^{*}-\phi^{*}\right\|_{E_{0}}\right) \leq \phi(0)
$$

and, since $(\varphi, \phi) \in \mathfrak{F}$, we infer that $\left\|\xi^{*}-\phi^{*}\right\|_{E_{0}} \leq 0$. Therefore, $\left\|\xi^{*}-\phi^{*}\right\|_{E_{0}}=0$ or, equivalently, $\xi^{*}=\phi^{*}$.

This result finishes the proof.

By Theorem 8, we obtain the following corollaries.

Corollary 9. Let $T: E_{0} \rightarrow E$ be a mapping such that there exists a pair of functions $(\varphi, \phi) \in \mathfrak{F}$ and $c \in[a, b]$ satisfying

$$
\varphi\left(\|T \xi-T \eta\|_{E}\right) \leq \phi\left(\|\xi-\eta\|_{E_{0}}\right)
$$

for any $\xi, \eta \in E_{0}$. If $R_{c}$ is topologically closed and algebraically closed with respect to difference, then $T$ has a unique PPF dependent fixed point in $R_{c}$.

Corollary 10. Let $T: E_{0} \rightarrow E$ be a mapping such that there exists a pair of functions $(\varphi, \phi) \in \mathfrak{F}$ and $c \in[a, b]$ satisfying

$$
\varphi\left(\|T \xi-T \eta\|_{E}\right) \leq \phi\left(\frac{\|\xi(c)-T \xi\|_{E}\|\eta(c)-T \eta\|_{E}}{1+\|T \xi-T \eta\|_{E}}\right)
$$

for any $\xi, \eta \in E_{0}$. If $R_{c}$ is topologically closed and algebraically closed with respect to difference, then $T$ has a unique PPF dependent fixed point in $R_{c}$.

The main result of [4] is Theorem 3 . Notice that the contractive condition appearing in this theorem

$$
\|T \xi-T \eta\|_{E} \leq \alpha\|\xi-\eta\|_{E_{0}}+\beta \frac{\|\xi(c)-T \xi\|_{E}\|\eta(c)-T \eta\|_{E}}{1+\|T \xi-T \eta\|_{E}}
$$

for any $\xi, \eta \in E_{0}$, where $\alpha, \beta \in[0,1)$ with $\alpha+\beta<1$ and $c \in[a, b]$, implies that

$$
\begin{aligned}
& \|T \xi-T \eta\|_{E} \\
& \leq(\alpha+\beta) \max \left\{\|\xi-\eta\|_{E_{0}}, \frac{\|\xi(c)-T \xi\|_{E}\|\eta(c)-T \eta\|_{E}}{1+\|T \xi-T \eta\|_{E}}\right\} \\
& \leq \max \left\{(\alpha+\beta)\|\xi-\eta\|_{E_{0}},\right. \\
& \left.\quad(\alpha+\beta) \frac{\|\xi(c)-T \xi\|_{E}\|\eta(c)-T \eta\|_{E}}{1+\|T \xi-T \eta\|_{E}}\right\}
\end{aligned}
$$

for any $\xi, \eta \in E_{0}$. This condition is a particular case of the contractive condition appearing in Theorem 8 with the pair of functions $(\varphi, \phi) \in \mathfrak{F}$ given by $\varphi=1_{[0, \infty)}$ and $\phi=(\alpha+\beta) 1_{[0, \infty)}$. Therefore, Theorem 3 is a particular case of the following corollary. 
Corollary 11. Let $T: E_{0} \rightarrow E$ be a mapping such that there exist real numbers $\alpha, \beta \in[0,1)$ with $\alpha+\beta<1$ and $c \in[a, b]$ satisfying

$$
\begin{aligned}
\|T \xi-T \eta\|_{E} \leq \max \left\{(\alpha+\beta)\|\xi-\eta\|_{E_{0}},\right. \\
\left.(\alpha+\beta) \frac{\|\xi(c)-T \xi\|_{E}\|\eta(c)-T \eta\|_{E}}{1+\|T \xi-T \eta\|_{E}}\right\}
\end{aligned}
$$

for any $\xi, \eta \in E_{0}$. If $R_{c}$ is topologically closed and algebraically closed with respect to difference, then $T$ has a unique PPF dependent fixed point in $R_{c}$.

Taking into account Example 5, we have the following corollary.

Corollary 12. Let $T: E_{0} \rightarrow E$ be a mapping such that there exist two functions $\varphi, \phi:[0, \infty) \rightarrow[0, \infty)$ and $c \in[a, b]$ such that

$$
\begin{aligned}
& \varphi\left(\|T \xi-T \eta\|_{E}\right) \leq \max \left\{\varphi\left(\|\xi-\eta\|_{E_{0}}\right)-\phi\left(\|\xi-\eta\|_{E_{0}}\right),\right. \\
& \varphi\left(\frac{\|\xi(c)-T \xi\|_{E}\|\eta(c)-T \eta\|_{E}}{1+\|T \xi-T \eta\|_{E}}\right) \\
&\left.-\phi\left(\frac{\|\xi(c)-T \xi\|_{E}\|\eta(c)-T \eta\|_{E}}{1+\|T \xi-T \eta\|_{E}}\right)\right\}
\end{aligned}
$$

for any $\xi, \eta \in E_{0}$, where $\varphi$ is a continuous and increasing function satisfying $\varphi(t)=0$ if and only if $t=0$, and $\phi$ is a nondecreasing function such that $\phi(t)=0$ if and only if $t=0$, and $\phi \leq \varphi$.

If $R_{c}$ is topologically closed and algebraically closed with respect to difference, then $T$ has a unique PPF dependent fixed point in $R_{c}$.

Corollary 12 has the following consequences.

Corollary 13. Let $T: E_{0} \rightarrow E$ be a mapping such that there exist two functions $\varphi, \phi:[0, \infty) \rightarrow[0, \infty)$ and $c \in[a, b]$ such that

$$
\varphi\left(\|T \xi-T \eta\|_{E}\right) \leq \varphi\left(\|\xi-\eta\|_{E_{0}}\right)-\phi\left(\|\xi-\eta\|_{E_{0}}\right)
$$

for any $\xi, \eta \in E_{0}$, where $\varphi$ is an increasing function and $\phi$ is a nondecreasing function and they satisfy $\varphi(t)=\phi(t)=0$ if and only if $t=0$, and $\varphi$ is continuous with $\phi \leq \varphi$. If $R_{c}$ is topologically closed and algebraically closed with respect to difference, then $T$ has a unique PPF dependent fixed point in $R_{c}$.

Corollary 13 can be considered as the version, in the context of PPF dependent fixed point theorems, of the following result about fixed point theorems which appears in [9].
Theorem 14 (see [9]). Let $(X, d)$ be a complete metric space and $T: X \rightarrow X$ a mapping satisfying

$$
\varphi(d(T x, T y)) \leq \varphi(d(x, y))-\phi(d(x, y))
$$

for $x, y \in X$, where $\varphi$ and $\phi$ satisfy the same conditions as in Corollary 13. Then $T$ has a unique fixed point.

Corollary 15. Let $T: E_{0} \rightarrow E$ be a mapping such that there exist two functions $\varphi, \phi:[0, \infty) \rightarrow[0, \infty)$ satisfying the same conditions as in Corollary 13 and $c \in[a, b]$ such that

$$
\begin{aligned}
\varphi\left(\|T \xi-T \eta\|_{E}\right) \leq & \varphi\left(\frac{\|\xi(c)-T \xi\|_{E}\|\eta(c)-T \eta\|_{E}}{1+\|T \xi-T \eta\|_{E}}\right) \\
& -\phi\left(\frac{\|\xi(c)-T \xi\|_{E}\|\eta(c)-T \eta\|_{E}}{1+\|T \xi-T \eta\|_{E}}\right)
\end{aligned}
$$

for any $\xi, \eta \in E_{0}$. If $R_{c}$ is topologically closed and algebraically closed with respect to difference, then $T$ has a unique PPF dependent fixed point in $R_{c}$.

Taking into account Example 6, we have the following corollary.

Corollary 16. Let $T: E_{0} \rightarrow E$ be a mapping such that there exist $\alpha \in S$ (see Example 6) and $c \in[a, b]$ satisfying

$$
\begin{aligned}
&\|T \xi-T \eta\|_{E} \leq \max \left\{\alpha\left(\|\xi-\eta\|_{E_{0}}\right)\|\xi-\eta\|_{E_{0}},\right. \\
& \alpha\left(\frac{\|\xi(c)-T \xi\|_{E}\|\eta(c)-T \eta\|_{E}}{1+\|T \xi-T \eta\|_{E}}\right) \\
&\left.\times \frac{\|\xi(c)-T \xi\|_{E}\|\eta(c)-T \eta\|_{E}}{1+\|T \xi-T \eta\|_{E}}\right\}
\end{aligned}
$$

for any $\xi, \eta \in E_{0}$. If $R_{c}$ is topologically closed and algebraically closed with respect to difference, then $T$ has a unique PPF dependent fixed point in $R_{c}$.

A consequence of Corollary 16 is the following result.

Corollary 17. Let $T: E_{0} \rightarrow E$ be a mapping such that there exists $\alpha \in S$ satisfying

$$
\|T \xi-T \eta\|_{E} \leq \alpha\left(\|\xi-\eta\|_{E}\right)\|\xi-\eta\|_{E_{0}}
$$

for any $\xi, \eta \in E_{0}$.

If $c \in[a, b]$ such that $R_{c}$ is topologically closed and algebraically closed with respect to difference, then $T$ has a unique PPF dependent fixed point in $R_{c}$.

Corollary 17 is the version, in the context of PPF dependent fixed point theorems, of the following result about fixed point theorems appearing in [10].

Theorem 18. Let $(X, d)$ be a complete metric space and $T$ : $X \rightarrow X$ a mapping satisfying

$$
d(T x, T y) \leq \alpha(d(x, y)) d(x, y)
$$

for any $x, y \in X$, where $\alpha \in S$. Then T has a unique fixed point. 


\section{Conflict of Interests}

The authors declare that there is no conflict of interests in the submitted paper.

\section{References}

[1] S. R. Bernfeld, V. Lakshmikantham, and Y. M. Reddy, "Fixed point theorems of operators with PPF dependence in Banach spaces," Applicable Analysis: An International Journal, vol. 6, no. 4, pp. 281-283, 1977.

[2] Z. Drici, F. A. McRae, and J. Vasundhara Devi, "Fixed-point theorems in partially ordered metric spaces for operators with PPF dependence," Nonlinear Analysis: Theory, Methods and Applications, vol. 67, no. 2, pp. 641-647, 2007.

[3] Z. Drici, F. A. McRae, and J. Vasundhara Devi, "Fixed point theorems for mixed monotone operators with PPF dependence," Nonlinear Analysis: Theory, Methods and Applications, vol. 69, no. 2, pp. 632-636, 2008.

[4] W. Sintunavarat and P. Kumam, "PPF dependent fixed point theorems for rational type contraction mappings in Banach spaces," Journal of Nonlinear Analysis and Optimization, vol. 4, no. 2, pp. 157-162, 2013.

[5] A. Kaewcharoen, "PPF dependent common fixed point theorems for mappings in Banach spaces," Journal of Inequalities and Applications, vol. 2013, no. 287, 2013.

[6] B. K. Dass and S. Gupta, "An extension of Banach contraction principle through rational expressions," Indian Journal of Pure and Applied Mathematics, vol. 6, pp. 1455-1458, 1975.

[7] D. S. Jaggi, "Some unique fixed point theorems," Indian J. Pure Appl. Math, vol. 8, pp. 223-230, 1977.

[8] M. Jleli, V. C. Rajié, B. Samet, and C. Vetro, "Fixed point theorems on ordered metric spaces and applications to nonlinear elastic beam equations," Journal of Fixed Point Theory and Applications, vol. 12, no. 1-2, pp. 175-192, 2012.

[9] P. N. Dhutta and B. S. Choudhury, "A generalization of contraction principle in metric spaces," Fixed Point Theory and Applications, vol. 2008, Article ID 406368, 2008.

[10] M. Geraghty, "On contractive mappings," Proceedings of the American Mathematical Society, vol. 40, pp. 604-608, 1973. 


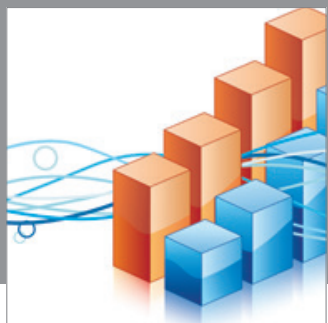

Advances in

Operations Research

mansans

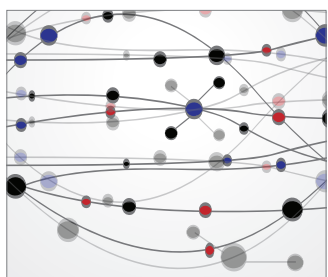

The Scientific World Journal
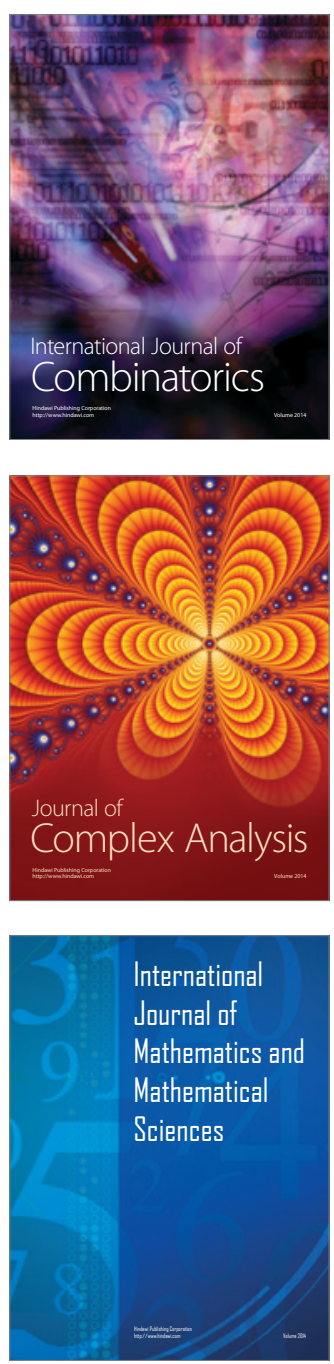
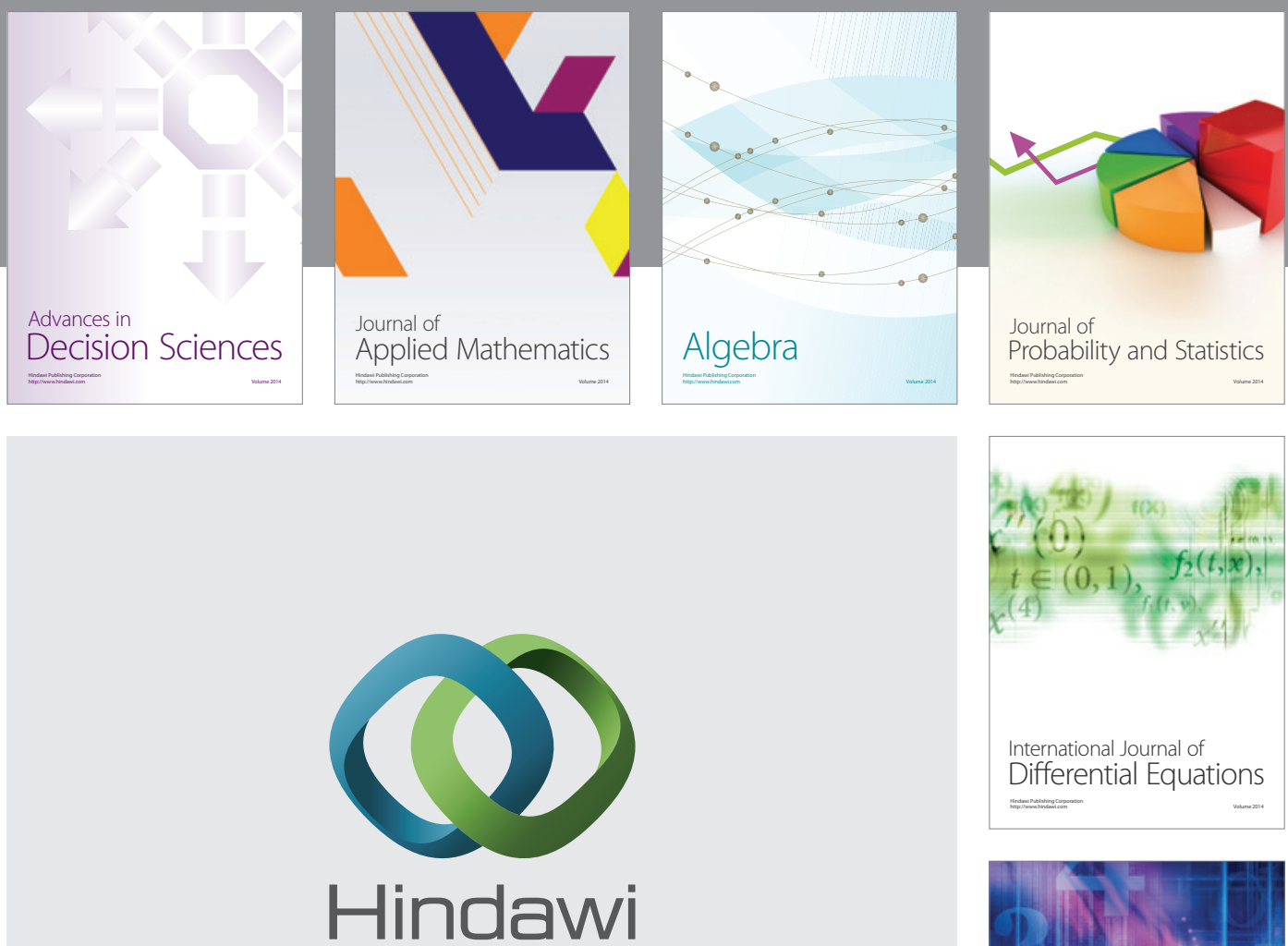

Submit your manuscripts at http://www.hindawi.com
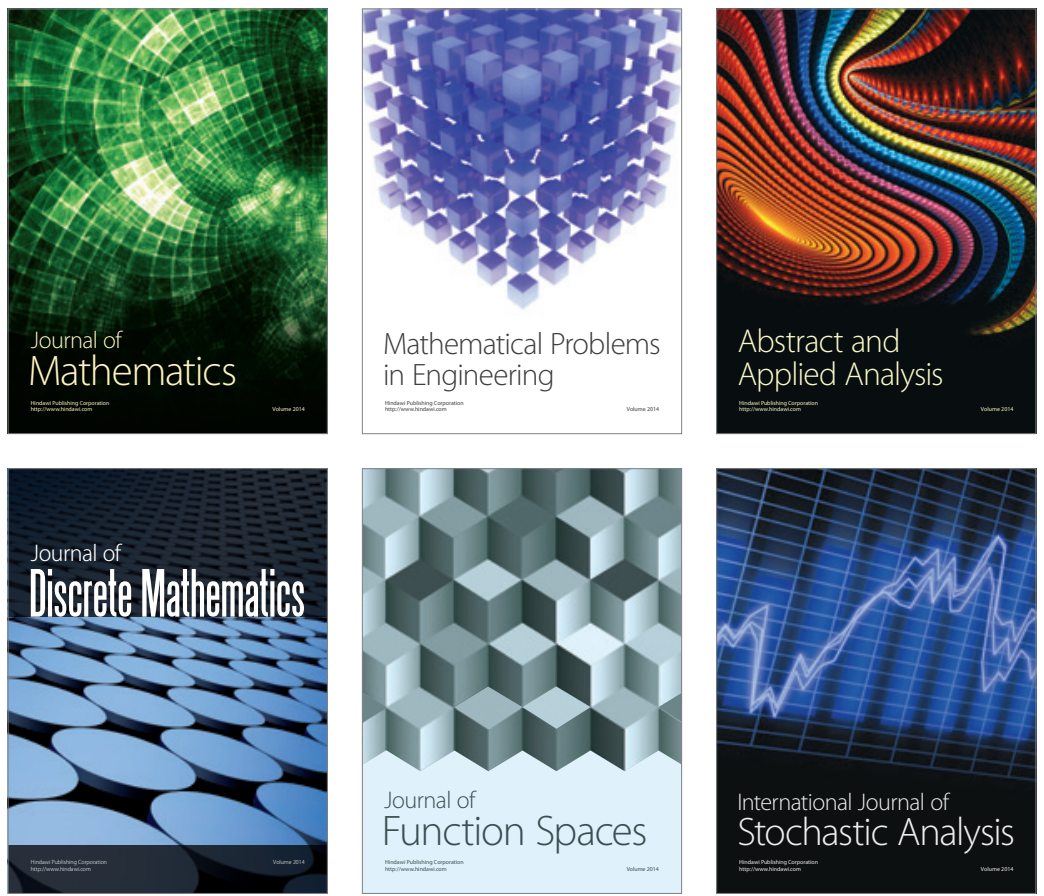

Journal of

Function Spaces

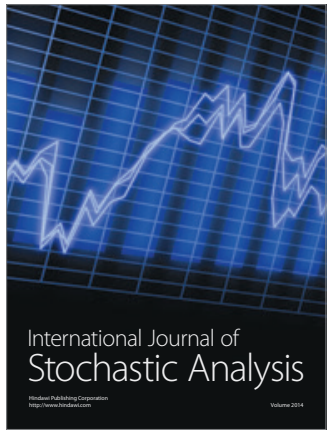

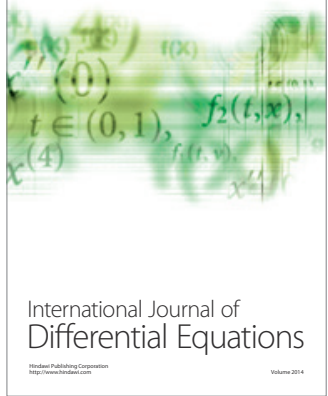
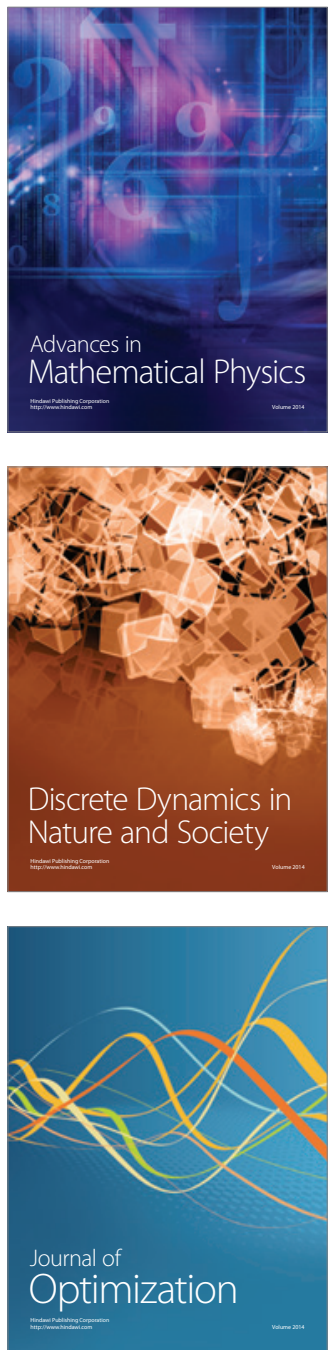